\title{
795 APV0603: A DUAL 4-1BB AND OX40 BISPECIFIC APPROACH UTILIZING ADAPTIRTM TECHNOLOGY DESIGNED TO DELIVER A CONDITIONAL T CELL/NK RESPONSE AGAINST SOLID TUMORS
}

Michelle Nelson*, Ashly Lucas, Rebecca Gottschalk, Catherine McMahan, Jane Gross, Hilario Ramos. Aptevo Therapeutics, Seattle, WA, United States

Background APVO603 is a dual targeting bispecific antibody for 4-1BB (CD137) and OX40 (CD134), engineered with Aptevo's ADAPTIRTM technology. We have previously shown that the distinct characteristics of APVO603 may enable conditional agonism of $4-1 \mathrm{BB}$ and OX40 only when cross-linked through engagement of the other receptor via cis and/or trans cellular interactions. Thus, APVO603 is designed with the potential to overcome both the on-target toxicity and limited efficacy observed with 4-1BB and OX40 monoclonal antibody treatment in the clinic.

Methods Genevestigator Software was used to analyze curated transcriptomic data for the expression profiles of OX40 and 4-1BB across select human heme and solid cancer patient sample data sets, as well as, non diseased tissue. Primary inducible Treg (iTreg) cells were sub-optimally stimulated with an antiCD3/CD28 antibody and cell proliferation was assessed using CFSE-labelled. Cytokines were measured using intracellular flow-based methods. For in vitro tumor lysis studies, activated $\mathrm{T}$ cells were co-cultured with Nuclight-labelled tumor cells expressing a tumor-associated antigen (TAA) and activated with TAA x CD3 bispecific protein. Live tumor cells were continually assessed using the Incucyte Live-Cell Analysis System and Cell-By-Cell Software Module.

Results OX40 and 4-1BB displayed distinct tumor expression profiles, however, several tumor indications were identified with high co-expression and may aid in identifying indications for the clinical development of APVO603. In vitro, APVO603 favored activation of effector $\mathrm{T}$ cell subsets and had minimal impact in augmenting iTreg cells proliferation, cytokine production or expression of effector-related molecules, despite the fact that a portion of the iTreg cells expressed OX40 and 41BB. The mechanistic activity of APVO603 resulted in dosedependent control of in vitro tumor growth when paired with a T-cell activating TAA x CD3 bispecific under standard conditions or those leading to $\mathrm{T}$ cell exhaustion. In preclinical assays using PBMCs sub-optimally stimulated with TAA $\mathrm{x}$ CD3, APVO603 enhanced TAA-expressing tumor cell lysis when compared to TAA x CD3 alone.

Conclusions APVO603 is a dual-agonistic bispecific antibody that augments the effector function of activated CD4+ and CD8 + T cells and NK cells, but not iTreg cells, in a dosedependent manner and reduces growth of tumors in vitro and in vivo. Further, mechanistic evaluation supports the ability of APVO603 to pair with T-cell modulating IO approaches to support a more fit $\mathrm{T}$ cell response and favorable TME. This preclinical data supports further development of APVO603, a promising immuno-oncology therapeutic with potential for benefit in hematologic and solid tumors.

http://dx.doi.org/10.1136/jitc-2021-SITC2021.795 